\title{
Temporal Invariance in SCA6 Is Related to Smaller Cerebellar Lobule VI and Greater Disease Severity
}

\author{
Basma Yacoubi, ${ }^{1}$ Agostina Casamento-Moran, ${ }^{1}$ Roxana G. Burciu, ${ }^{1}$ S.H. Subramony, ${ }^{2}$ David E. Vaillancourt, ${ }^{1,2,3}$ \\ and Evangelos A. Christou ${ }^{1,2,4}$ \\ ${ }^{1}$ Department of Applied Physiology and Kinesiology, ${ }^{2}$ Department of Neurology, ${ }^{3}$ Department of Biomedical Engineering, and ${ }^{4}$ Department of Physical \\ Therapy, University of Florida, Gainesville, Florida 32611
}

Regulating muscle force and timing are fundamental for accurate motor performance. In spinocerebellar ataxia type 6 (SCA6), there is evidence that individuals have greater force dysmetria but display better temporal accuracy during fast goal directed contractions. Here, we test whether greater temporal accuracy occurs in all individuals with SCA6, and can be explained by lesser temporal variability. Further we examine whether it is linked to disease severity and specific degenerative changes in the cerebellum. Nineteen human participants with SCA6 (13 woman) and 18 healthy controls performed fast goal-directed ankle dorsiflexion contractions aiming at a spatiotemporal target. We quantified the endpoint control of these contractions, gray matter (GM) integrity of the cerebellum, and disease severity using the International Cooperative Ataxia Rating Scale (ICARS). SCA6 individuals exhibited lower temporal endpoint error and variability than the healthy controls $(p=0.008)$. Statistically, SCA6 clustered into two distinct groups for temporal variability. A group with low temporal variability ranging from 10 to $19 \%$ (SCA6a) and a group with temporal variability similar to healthy controls (SCA6b; $19-40 \%) . S C A 6 a$ exhibited greater disease severity than SCA6b, as assessed with ICARS $(p<0.001)$. Lower temporal variability, which was not associated with disease duration $\left(R^{2}=0.1, p>0.2\right)$, did correlate with both greater ICARS $\left(R^{2}=0.3\right)$ and reduced GM volume in cerebellar lobule VI $\left(R^{2}=0.35\right)$. Other cerebellar lobules did not relate to temporal variability. We provide new evidence that a subset of SCA6 with greater loss of GM in cerebellum lobule VI exhibit temporal invariance and more severe ataxia than other SCA6 individuals.

Key words: cerebellum; dysmetria; MRI; spinocerebellar ataxia; temporal variability

Significance Statement

Variability is an inherent feature of voluntary movement, and traditionally more variability in the targeted output infers impaired performance. For example, cerebellar patients present exacerbated temporal variability during multijoint movements, which is thought to contribute to their motor deficits. In the current work, we show that in a subgroup of spinocerebellar ataxia type 6 individuals, temporal variability is lower than that of healthy controls when performing single-joint fast-goal directed movements. This invariance related to exacerbated atrophy of lobule VI of the cerebellum and exacerbated disease severity. The relation between invariance and disease severity suggests that pathological motor variability can manifest not only as an exacerbation but also as a reduction relative to healthy controls.

\section{Introduction}

Spinocerebellar ataxia type 6 (SCA6) is a slow progressing neurodegenerative disorder of the cerebellum (Zhuchenko et al.,

\footnotetext{
Received June 28, 2019; revised Dec. 6, 2019; accepted Dec. 13, 2019.

Author contributions: B.Y., D.E.V., and E.A.C. designed research; B.Y., A.C.-M., R.G.B., and S.H.S. performed research;B.Y., A.C.-M., R.G.B., D.E.V., and E.A.C. analyzed data; B.Y. and E.A.C. wrote the first draft of the paper; B.Y., A.C.-M., R.G.B., S.H.S., D.E.V., and E.A.C. edited the paper.

This work was supported by the National Institutes of Health/National Institute of Neurological Disorders and Stroke (Grants R21 NS094946 and R21 NS093695 to D.E.V. and E.A.C.).

The authors declare no competing financial interests.

Correspondence should be addressed to Evangelos A. Christou at eachristou@ufl.edu.

https://doi.org/10.1523/JNEUROSCI.1532-19.2019

Copyright $@ 2020$ the authors
}

1997; Solodkin and Gomez, 2012). One cardinal symptom of SCA6 is dysmetria, which is defined as the inability to perform accurate movements (Holmes, 1917, 1922; Manto, 2009; Casamento-Moran et al., 2015). Further, there is abundant evidence that cerebellar atrophy, as occurring in SCA6, impairs temporal control (Bastian et al., 1996; Topka et al., 1998; Babin-Ratté et al., 1999; Hore et al., 2002; Ivry and Keele, 1989; Nowak et al., 2002; Spencer et al., 2003; Grube et al., 2010; Becker et al., 2015; Paquette et al., 2017). Nonetheless, we have recently shown that some SCA6 patients exhibit greater temporal accuracy during single-joint fast goal-directed movements (Casamento-Moran et al., 2015), presumably due to lower temporal variability. This 
lower temporal variability may be a consequence of a cerebellar lobule VI change (Baer et al., 2015). Thus, in this study, we address the following question: Do SCA6 patients with reduced temporal variability exhibit distinct structural changes in cerebellar lobule VI and consequently reduced disease severity?

Temporal control of action has consistently been linked to the cerebellum (Bares et al., 2019). A great number of studies dealing with temporal control of action are based on cerebellar patients who exhibit increased temporal variability during repetitive finger tapping and circle drawing (Ivry and Keele, 1989; Spencer et al., 2003) and impaired judgment of temporal intervals between sensory stimuli (Ivry and Keele, 1989; Grube et al., 2010; Paquette et al., 2017). In addition, they exhibit impaired time control during arm reaching (Bastian et al., 1996; Topka et al., 1998), object intercepting (Babin-Ratté et al., 1999; Nowak et al., 2002), and overarm throwing (Hore et al., 2002, 2005; Hore and Watts, 2005; Becker et al., 2015). Recently, we characterized dysmetria in SCA6 individuals using single-joint fast goal-directed movements that require spatial and temporal control (CasamentoMoran et al., 2015). Similar to findings using multijoint movement tasks, we have shown that SCA6 exhibit greater spatial dysmetria and endpoint variability than healthy controls. In contrast to our expectations, however, we found that some SCA6 exhibited significantly greater temporal control relative to healthy controls. This surprising finding poses the following question: How does greater temporal control in fast single-joint movements relate to cerebellar pathology and functional impairment in individuals with SCA6?

The greater temporal control during single-joint movements in SCA6 patients may be due to low temporal variability (invariance), which could signify a specific pattern of cerebellar atrophy. Indeed, a study on healthy musicians links reduced temporal variability and better motor performance to smaller cerebellar lobule VI (Baer et al., 2015). In both healthy musicians and SCA6, a smaller lobule VI could result in low temporal variability of the contributing single joints. Given that complex motor actions result from the coordination (amplitude and relative timing) of individual single joints, reduced temporal variability of single joints could result in less variable multijoint performance for SCA6. Alternatively, if this reduced temporal variability relates to a smaller lobule VI, it may be a signature of a greater cerebellar degeneration in SCA6 and could be associated with impaired performance of complex motor actions. Regardless, the relation of greater temporal variability in single joints to the disease severity and specific cerebellar changes in SCA6 individuals remain unresolved.

Thus here, we used single joint, self-initiated discrete fast goaldirected isometric contractions in a SCA6 cohort $(N=19)$ to characterize temporal control in relation to regional structural changes in the cerebellum and disease severity. We test the hypothesis that SCA6 individuals with reduced temporal variability will exhibit distinct structural changes in cerebellar lobule VI and reduced disease severity.

\section{Materials and Methods}

Standard protocol approvals, registrations, and patient consents Our study procedures were designed in accordance of the Declaration of Helsinki and were approved by the Institutional Review Board at the University of Florida. All participants gave their written informed consent before participating in the study.

\section{Participants}

Nineteen individuals genetically confirmed with SCA6 (62 \pm 9.75 years.; 13 females) and 18 age-matched healthy controls (60.5 \pm 10.1 years.; 10 females) volunteered to participate in this study. All participants were right-footed (Elias et al., 1998). Inclusion criteria for participants with SCA6 were DNA diagnosis of SCA6, phenotype consistent with the DNA diagnosis, ability to walk $7 \mathrm{~min}$, age between 21 and 85 years, MRI compatible, and no pregnancy. Inclusion criteria for control participants were healthy with no neurological impairments, age between 21 and 85 years, MRI compatible, and no pregnancy.

\section{Experimental approach}

Participants performed all testing procedures within a day. There were two distinct $2 \mathrm{~h}$ sessions. They performed the MRI testing in the morning and the motor control session in the afternoon. Within the motor control session, each participant performed the following four procedures: (1) maximal voluntary contraction (MVC) with ankle dorsiflexion and plantarflexion (pretask); (2) three to five goal-directed contractions practice trials at a different target from the actual target; (3) 50 goal-directed contractions trials (five blocks of 10 trials) with ankle dorsiflexion at $15 \%$ of the MVC; and (4) posttask MVC as in procedure 1.

\section{Experimental setup}

The experimental set up followed in this study was described in detail previously (Casamento-Moran et al., 2015) (Fig. 1A). In brief, participants sat with their left hip joint flexed to $\sim 90^{\circ}$ with $10^{\circ}$ abduction, the knee flexed to $\sim 90^{\circ}$, and the ankle plantarflexed to $\sim 15^{\circ}$. The left foot rested on a customized foot device with an adjustable footplate and was strapped over the metatarsals. We positioned the axis of rotation of the device in line with the axis of rotation of the left ankle to allow only dorsiflexion and plantarflexion.

\section{Equipment}

We measured the forces exerted during contraction using a force transducer (model MB-100; Interface) located in parallel with the force direction on the customized foot device. We sample the ankle force signal at $1000 \mathrm{~Hz}$ with a NI-DAQ card (model USB6210; National Instruments), and stored them on a personal computer. We used a 32-inch monitor (Sync Master 320MP-2; Samsung Electronics) located $1.25 \mathrm{~m}$ away and at eye level to display task related directions and visual feedback of the performance after each trial using a custom-written program in MATLAB (The MathWorks).

\section{Clinical assessments}

Strength. Participants performed a maximum voluntary isometric contraction (MVC) task before the goal directed contraction task (preMVC) to calculate the target force for each participant. Participants repeated the same task (post-MVC) to determine whether the experimental protocol induced fatigue. To perform the MVC task, participants increased force to their maximum and maintained it for three seconds and maintained it for $3 \mathrm{~s}$. They exerted 3-5 MVCs or until two MVC trials were within $5 \%$ of each other.

Endpoint control. Participants performed fast aiming isometric contractions with ankle dorsiflexion. They aimed to achieve 15\% MVC in $180 \mathrm{~ms}$ to match a spatiotemporal target displayed on the computer monitor. We chose $180 \mathrm{~ms}$ based on published values of the minimum time required for on-line corrections to unexpected perturbations during goal-directed movements (Carlton, 1981; Prablanc and Martin, 1992; Desmurget and Grafton, 2003; Saunders and Knill, 2004; Grafton and Tunik, 2011; Oostwoud Wijdenes et al., 2011). Importantly, from our experience with the task used in this study, a $180 \mathrm{~ms}$ target requirement yields smooth trajectories to the target (no corrective submovements) consistant with an open-loop control.

The dorsiflexion task was divided in three consecutive phases: (1) GET READY (red target on the monitor for $2 \mathrm{~s}$ ), (2) CONTRACT (green target on the monitor for $3 \mathrm{~s}$ ), and (3) FEEDBACK ( $5 \mathrm{~s}$ ). The participants performed the goal-directed contraction at their convenience during the three second-window of the CONTRACT phase, therefore, this was not a reaction time task. The recording of the task began when the participant initiated the contraction within the $3 \mathrm{~s}$ of the CONTRACT phase. We 
A

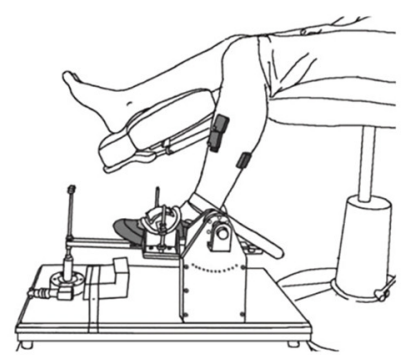

B

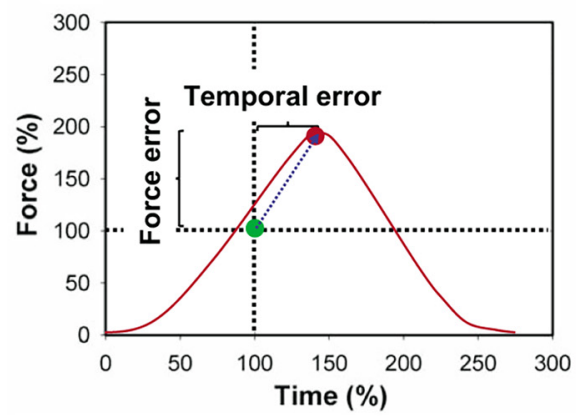

C

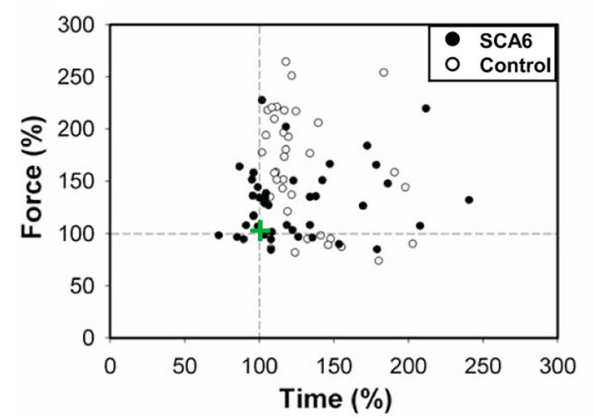

D

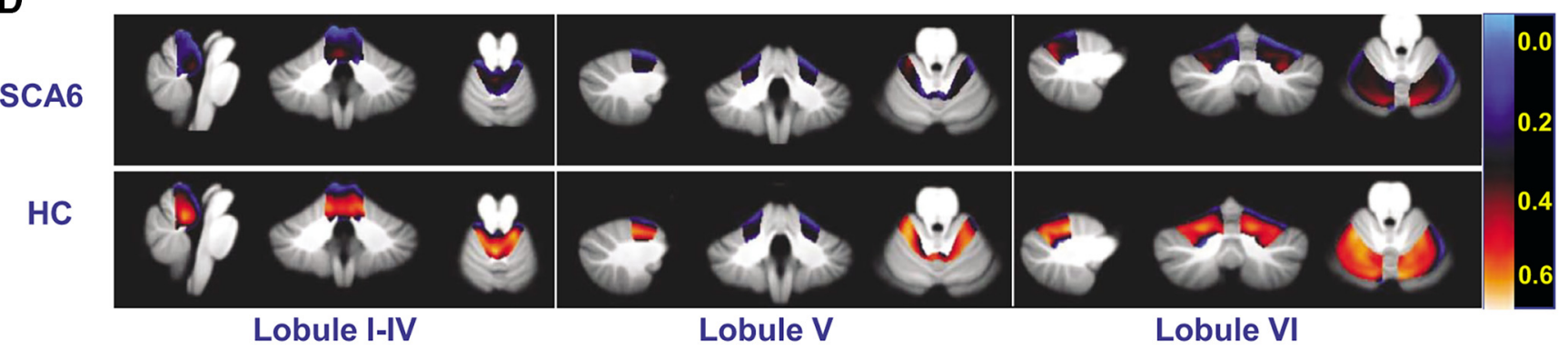

Figure 1. Experimental paradigm to characterize endpoint control and associated cerebellar attributes in SCA6. A, Subjects performed 50 goal-directed ankle dorsiflexion contractions with the left foot. $\boldsymbol{B}$, Force and time-to peak force errors were normalized to the force and time targets (15\% MVC and $180 \mathrm{~ms}$, respectively). $\boldsymbol{C}$, Representative endpoints of 50 goal-directed trials for a SCA6 (open circles) and an age-matched control participant (closed circles) relative to the target (in green at 100\%, 100\%). D, Mean GM density within lobules I-IV, V, and VI for SCA6 patients and healthy controls. As expected, the plot indicates greater density of GM tissue in all lobules in the control group compared with SCA6. VBM measures are overlaid on the SUIT template.

provided the participants with visual feedback of their contraction trace relative to the targeted force-time endpoint (Fig. 1). The visual gain was kept constant at $1^{\circ}$ (visual angle) for all trials. Participants performed five blocks of 10 trials for a total of 50 trials.

All patients received a comprehensive neurological evaluation to assess neurological and functional impairments and confirm consistency with the genetic diagnosis.

Disease severity. We quantified disease severity of SCA6 using the International Cooperative Ataxia Rating Scale (ICARS), and the Scale for Assessment and Rating of Ataxia (SARA) (Trouillas et al., 1997; Storey et al., 2004; Saute et al., 2012). The ICARS is a 100-point clinical rating scale, divided into 4 subscales: posture and gait disturbance ( 7 items, 34 points); limb ataxia ( 7 items, 52 points); speech disorders ( 2 items, 8 points); and oculomotor disorder ( 3 items, 6 points). The SARA is a 40 points performance based scale, subdivided into eight items (gait, stance, sitting, speech disturbance, finger chase, nose-finger test, fast alternating hand movements, heel-shin slide. SARA and ICARS scores were highly correlated $\left(R^{2}=0.92, p<000.1\right)$, thus only ICARS scores were used for this study. Higher value in ICARS and SARA indicate greater severity.

Cognition and depression. Cognitive ability was measured using the Montreal Cognitive Assessment test (MoCA) (Nasreddine et al., 2005), and anxiety and depression were measured using the Beck Depression Index (Beck et al., 1961).

\section{Data analysis}

Endpoint error and variability. For each trial, we quantified force and temporal endpoint error and variability. We quantified force error as the peak force relative to the targeted peak force.

Force error was quantified as follows:

$$
\text { Force error }(\%)=\frac{\mid \text { Peak force }- \text { Target peak force } \mid}{\text { Target peak force }} * 100
$$

We quantified temporal error as time to peak force relative to the targeted time to peak force.

Temporal error was quantified as follows:
Temporal error $(\%)=$

$$
\frac{\mid \text { Time to peak force }- \text { Target time to peak force } \mid}{\text { Target time to peak force }} * 100
$$

We eliminated outlier trials, defined as the peak force or time to peak force exerted below fifth and above $95^{\text {th }}$ percentile of all the trials.

We also quantified the endpoint variability in peak force and time to peak force across trials. The trial-to-trial variability was quantified using the coefficient of variation $(\mathrm{CV})$ for peak force and time to peak force.

Force variability was quantified as follows:

$$
\text { Force variability }(\%)=\frac{\mid S D \text { of peak force } \mid}{\text { mean peak force }} * 100
$$

Temporal variability was quantified as follows:

$$
\text { Temporal variability }(\%)=\frac{\mid S D \text { of Time to peak force } \mid}{\text { mean Time to peak force }} * 100
$$

\section{MRI data acquisition and processing}

For each participant, a high-resolution $3 \mathrm{D} \mathrm{T}_{1}$-weighted MRI scan was acquired using a $3 \mathrm{~T}$ Philips Achieva scanner equipped with a 32-channel SENSE head coil. The scan had the following parameters: repetition time $(\mathrm{TR})=8.2 \mathrm{~ms}$, echo time $(\mathrm{TE})=3.7 \mathrm{~ms}$, flip angle $=8^{\circ}$, field of view $(\mathrm{FOV})=240 \mathrm{~mm}^{2}$, voxel size $=1 \mathrm{~mm}$ isotropic with no gap between slices (slice no. = 170). Quality control of the MRI scans was performed by examining each image for anatomical abnormalities and scanner artifacts.

\section{MRI data analysis: voxel-based morphometry (VBM)}

Data preprocessing was performed in SPM8 running in MATLAB R2013a, using the VBM8 toolbox (http://www.neuro.uni-jena.de/vbm/) and the SUIT toolbox (http://www.diedrichsenlab.org/imaging/suit. $\mathrm{htm}$ ). The following procedures were implemented: setting the origin to the anterior commissure; segmentation into gray matter (GM), white 
A
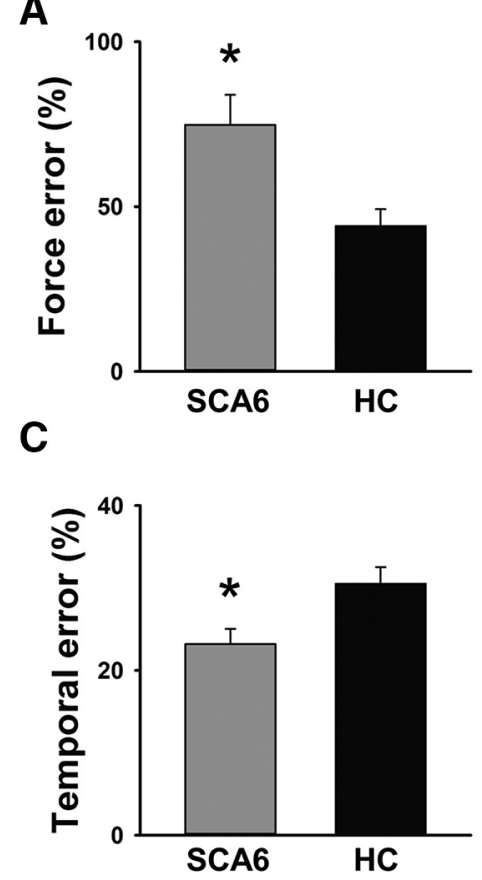

B
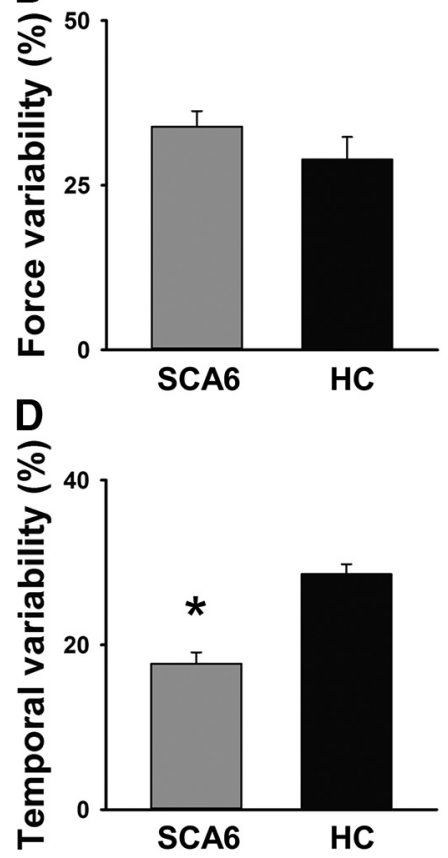

Figure 2. Error and variability of temporal and force endpoint of ankle dorsiflexion contractions for SCA6 and healthy controls. Shown are force error $(\boldsymbol{A})$, force variability $(\boldsymbol{B})$, temporal error $(\boldsymbol{C})$, and temporal variability $(\boldsymbol{D})$ for the five blocks of 10 fast goal-directed contractions. Force error, temporal error, and temporal variability were significantly lower for SCA6 participants compared with healthy controls as indicated by the asterisk ( $p=0.001)$.

matter (WM), and CSF, transformation of the GM scan to a standard template using DARTEL. For normalization of the cerebellum we used the SUIT template. The SUIT is optimal because it preserves the anatomical detail of the cerebellum (Diedrichsen, 2006). To correct for the local expansion or contraction inherent to the normalization process, GM scans were modulated. Next, the GM scans were smoothed with a Gaussian kernel with a full width at half maximum (FWHM) size of $8 \mathrm{~mm}$ for the cerebrum and $4 \mathrm{~mm}$ for the cerebellum. A region of interest (ROI) approach was used to look at group differences in the amount of GM in cerebellum. For quantifying GM in the cerebellar lobules, the SUIT probabilistic atlas was used (Diedrichsen, 2006). This process resulted in GM measurements for the 10 bilateral lobules (I-X right and I-X left; lobules I-IV are combined into one measure, and lobule VII is divided into VIIB, Crus I and Crus II; lobule VIII is divided into VIIIA and VIIIB) and vermis lobules VI-X.

\section{Statistical analysis: dependent variables}

The dependent variables were as follows: Disease severity: ICARS and ICARS sub-scale scores. Endpoint control: force error, temporal error, force variability and temporal variability (\%). Participant characteristics: age, ankle dorsiflexion strength, disease duration, and MoCA. Cerebellar GM volume: cerebellar lobule I-IV, V, VI, CrusI, CrusII, VIIb, VIIIa, VIIIb, IX, X and vermis lobules VI, CrusI, CrusII, VIIb, VIIIa, VIIIb, IX, X.

To compare the dependent variables between SCA6 and healthy controls we used independent $t$ tests. For multiple comparisons we FDR-corrected and interpreted significance at $p<0.05$ FDRcorrected (Benjamini and Hochberg procedure) (Benjamini and Hochberg, 1995). Similarly, we compared two subgroups within SCA6 (SCA6a vs SCA6b) that we identified using k-mean clustering of temporal variability data (see results for further explanation of these two subgroups). Based on temporal variability, it was clear that 2 subgroups of SCA6 represented the empirical data (see Fig. 3B). One group with similar temporal variability to healthy controls and a second group with lower temporal variability than healthy controls (see Fig. 3B). The empirical distinction of two SCA6 groups based on temporal variability was verified statistically with a one-way ANOVA (see Results).
We used stepwise linear regression analysis to establish statistical models that could predict temporal variability in SCA6 (dependent variable) from regional cerebellar volume (independent variables). Using similar methodology, we predicted disease severity (ICARS; dependent variable) from participant characteristics, endpoint control, and regional cerebellar GM volume (independent variables). The goodness of fit of each model was given by the squared multiple correlations $\left(R^{2}\right)$ and Durbin Watson statistic (DW) (Green and Salkind, 2004). All statistical analyses were performed with the IBM statistics 25.0 statistical package. The $\alpha$ level for all statistical tests was a corrected $\mathrm{p}$ value equal to 0.05 . Data are reported as means \pm SD in the text and as means \pm SE in figures. Only the significant main effects and interactions are presented, unless otherwise noted.

\section{Data availability}

Anonymized data not published within this article will be made available by request from any qualified investigator.

\section{Results}

\section{Endpoint performance: force and} temporal control

To characterize control of endpoint during fast goal-directed contractions we quantified force error, force variability, temporal error, and temporal variability of the endpoint. We found that SCA6 individuals exhibited greater force error ( $p=0.03$; Fig. $2 A$ ) but lower temporal error $(p=0.03$; Fig. $2 C)$ and lower temporal variability $(p<0.001$; Fig. $2 D)$ relative to healthy controls. The temporal error and temporal variability were associated $\left(R^{2}=0.44, p=0.001\right)$ as well as the force error and force variability $\left(R^{2}=0.27, p=0.02\right)$ but there were no associations between force endpoint variability and temporal endpoint variability.

We examined the distribution of force and temporal endpoints in SCA6 and healthy control participants (Fig. 3A). SCA6 participants grouped into two distinct groups based on temporal variability of endpoint, those with temporal variability $<19 \%$ (SCA6a) and those with variability $>19 \%$ (SCA6b). This grouping was specific to temporal control as there was no evident grouping based on force variability (Fig. 3B). One-way ANOVAs statistically verified that SCA6a exhibited significantly lower temporal variability than SCA6b and healthy controls $(F=48.93, p<$ $0.001)$, whereas force variability was similar among the 3 groups $(F=0.72, p>0.49)$. Thus the lower temporal variability for the SCA6 group as a whole was driven by a subset of SCA6 individuals (12 individuals, 63\%) exhibiting temporal variability ranging from 10 to $19 \%$, while the remaining seven SCA6 individuals and healthy controls ranged from 19 to $35 \%$.

The SCA6a (temporally invariant group) exhibited lower temporal error than SCA6b (20.06 $\pm 7.60 \%$ vs $28.59 \pm 6.10 \%$; $p=0.008)$ even though their average endpoint performance was not different (i.e., both groups were on average equally close to the target). Specifically, the average peak force $(35.18 \pm 22.63 \mathrm{~N}$ vs $46.69 \pm 25.15 \mathrm{~N} ; p=0.17)$ and average time to peak force $(206.93 \pm 24.95 \mathrm{~ms}$ vs $222.84 \pm 21.36 \mathrm{~ms}$; $p=0.08$ ) were not statistically different for SCA6a and SCA6b. Therefore, the apparent greater temporal control (lower tem- 
A

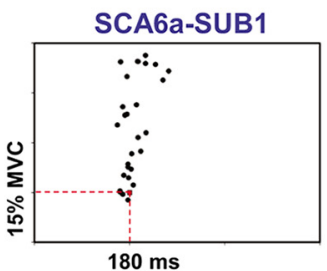

SCA6b-SUB1

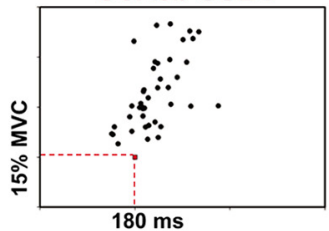

HC-SUB1

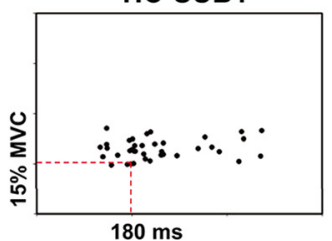

B

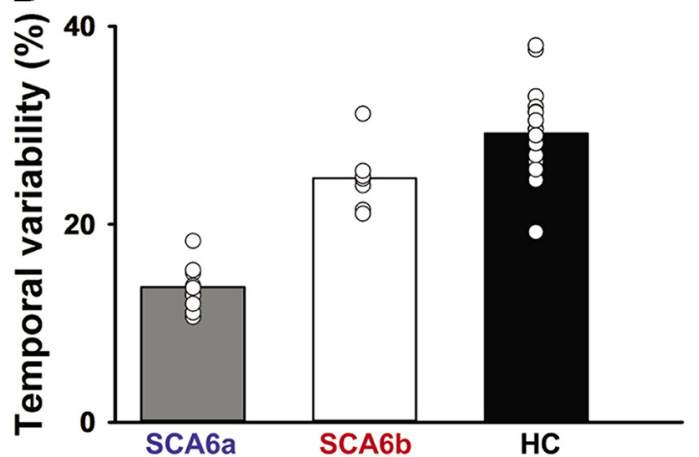

SCA6a-SUB2

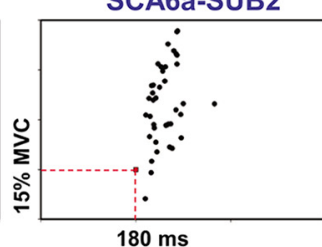

SCA6b-SUB2

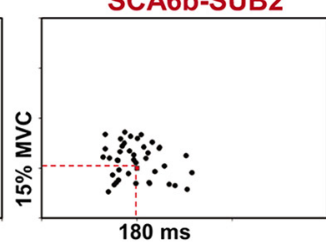

HC-SUB2

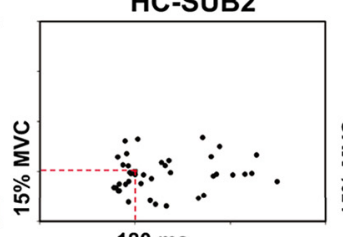

$80 \mathrm{~ms}$
SCA6a-SUB3

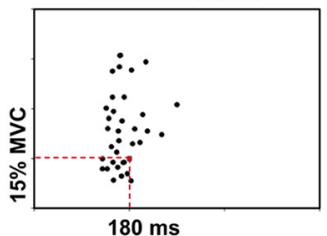

SCA6b-SUB3

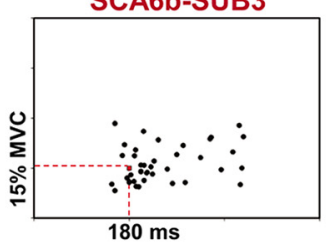

HC-SUB3
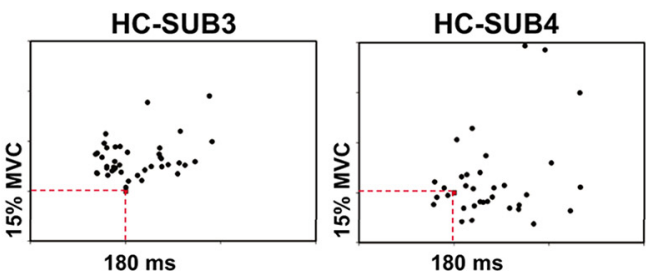

SCA6a-SUB4

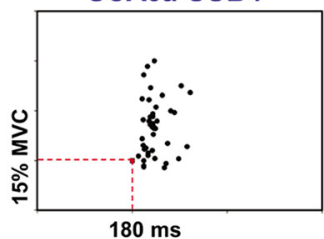

SCA6b-SUB4

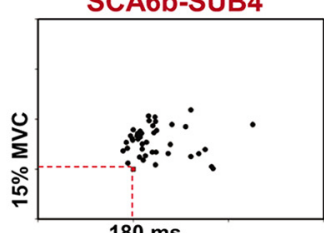

$180 \mathrm{~ms}$

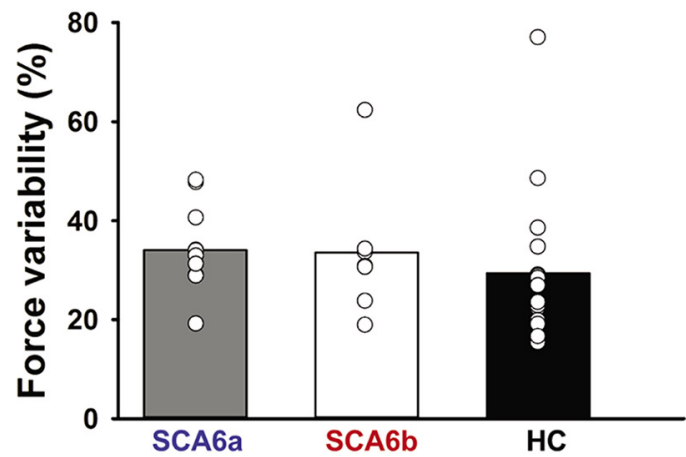

Figure 3. Distinct temporal control behaviors among SCA6 individuals. k-means cluster analysis separated SCA6 into two groups, SCA6a and SCA6b based on their temporal variability. $A$, Distribution of endpoints for four representative SCA6a (SCA6a-SUB1, SCA6a-SUB2, SCA6a-SUB3, and SCA6a-SUB4), SCA6b (SCA6b-SUB1, SCA6b-SUB2, SCA6b-SUB3, and SCA6b-SUB4) and healthy control (HC1-SUB1, HC-SUB2, HC-SUB3, and SUB4-HC) participants (top, middle, and bottom, respectively). Over the five blocks, SCA6a individuals exhibited lower temporal variability, whereas SCA6b individuals exhibited temporal variability comparable to healthy controls. $\boldsymbol{B}$, Temporal variability for the SCA6a group was $<19 \%$ and temporal variability of the SCA6b and $\mathrm{HC}$ group was $>19 \%$. Individuals in the SCA6b group had similar variability to healthy controls (left). Force variability was similar for the three groups (right).

poral error) in SCA6a reflects their reduced temporal variability compared with SCA6b.

\section{Temporal endpoint invariance: SCA6 characteristics and disease severity}

We compared participant characteristics (age, disease duration, strength, cognitive capacity) between SCA6a and SCA6b and found no statistical differences $(p>0.5)$ (Fig. $4 A)$. The ICARS however, was significantly greater for the SCA6a (temporally invariant group) than SCA6b $(p=0.04)$ (higher value in ICARS indicates greater severity). Of the ICARS subscales, limb ataxia scores $(p=0.04)$, postural and gait disturbances scores $(p=0.04)$, and oculomotor disorders scores $(p=0.04)$ were significantly greater for SCA6a; whereas the dysarthria score was not different $(p>0.3)$ (Fig. 4B). Therefore, SCA6a exhibited greater disease severity than SCA6b with similar participant characteristics.

\section{Temporal endpoint invariance and cerebellar GM volume}

As expected, the GM volume of all tested cerebellar regions was significantly different between SCA6 and healthy controls $(p=$
$0.004)$. We predicted the temporal variability from GM volume of cerebellar lobules I-IV, V, VI, CrusI, CrusII, VIIb, VIIIa, VIIIb, IX, and X and vermis lobules VI, CrusI, CrusII, VIIb, VIIIa, VIIIb, IX, and $\mathrm{X}$, using a stepwise linear regression model. Of all the cerebellar regions tested, the GM volume for lobule VI was the only predictor of temporal variability in SCA6 $\left(R^{2}=0.35\right.$; Fig. $5 A)$; with lower temporal variability being associated with lower GM volume in lobule VI.

We then compared GM volume of the two SCA6 groups. We found that the time invariant SCA6a group showed less GM volume in lobule VI ( $p=0.04$; Fig. $5 B, C)$. Therefore, the temporal invariance in SCA6a, which explained their better overall accuracy performance, relates to a reduced GM volume of cerebellar lobule VI.

\section{Predicting disease severity in SCA6}

We used a stepwise multiple linear regression to derive statistical models that predict disease severity (ICARS total) from participants' characteristics, endpoint performance, and cerebellar GM. 
A
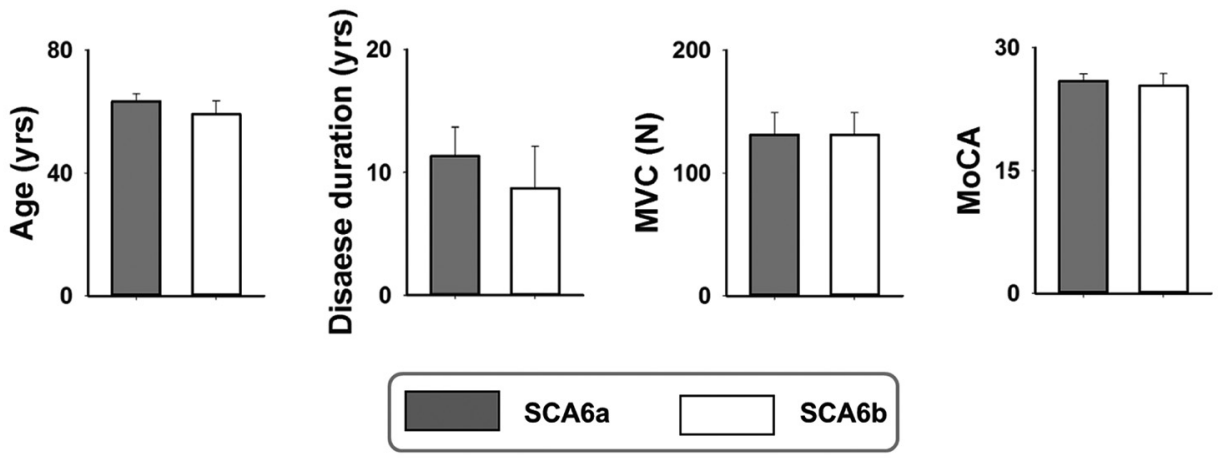

B
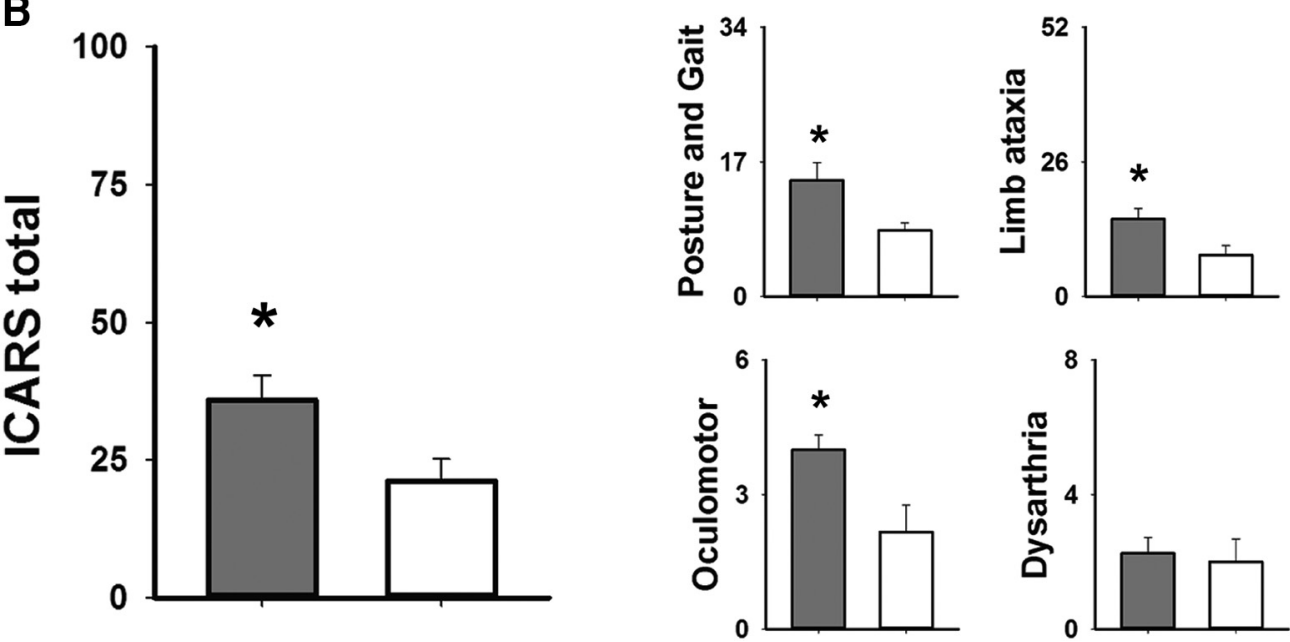

Figure 4. Characteristics and disease severity in the two SCA6 groups differing in temporal control. $A$, There were no significant differences between the two groups for MoCA (cognitive assessment; SCA6a $25.92 \pm 2.97$; SCA6b 25.33 \pm 3.67 ), disease duration (SCA6a 11.33 years. \pm 8.16 ; SCA6b $8.71 \pm 8.99$ ), age (SCA6a 63.33 years. $\pm 08.63 ;$ SCA6b 59.14 years. \pm 11.65 ), and strength (MVC) (SCA6a 131.23 N $\pm 61.96 ;$ SCA6b 191.74 N \pm 99.53$)(p>0.5)$. B , Disease severity (ICARS total) was greater for the SCA6a (temporally invariant group) than SCA6b ( $p=0.04)$ (left) (the greater the ICARS score the greater the disease severity). Of the four ICARS subscales, limb ataxia scores ( $p=0.04)$, postural and gait disturbances scores $(p=0.04)$, and oculomotor disorders scores $(p=0.04)$ were significantly greater for the SCA6a; whereas the dysarthria score was not different $(p>0.3)$ (right). Asterisks indicates significance $(p<0.05)$.

A

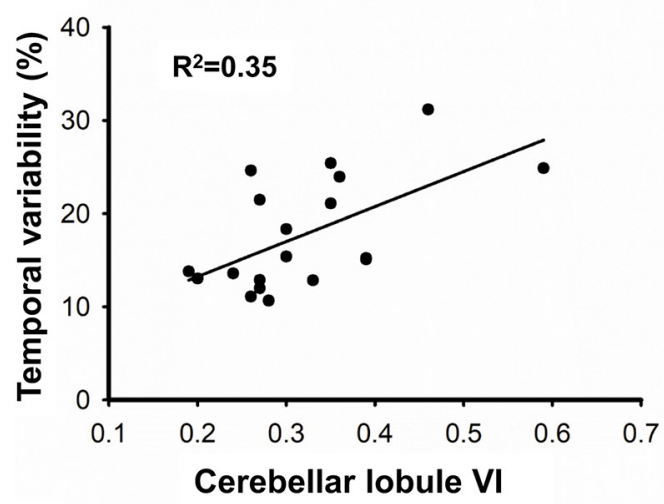

B

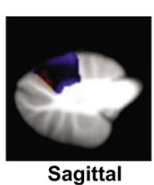

SCA6a

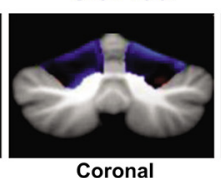

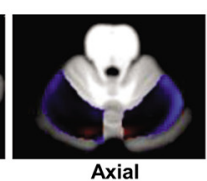
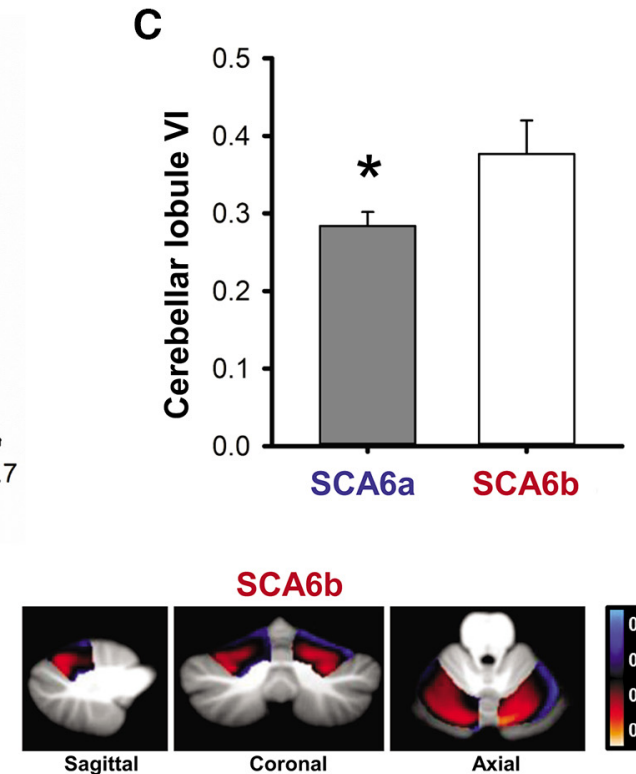

SCA6b
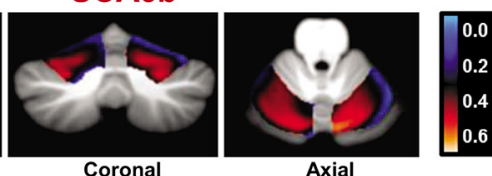

Coronal

Axial

Figure 5. Prediction of low temporal variability from GM volume in the cerebellum. $A$, Reduced time variability was predicted from lower $\mathrm{GM}$ volume in cerebellar lobule VI $\left(R^{2}=0.36 ; p=\right.$ 0.007). B, Mean GM density within lobules VI for SCA6a and SCA6b. The plot indicates that GM volume in cerebellar lobule VI was smaller for SCA6a compared with SCA6b ( $p=0.05)$. C, VBM measures are overlaid on the SUIT template. Asterisk indicates significance $(p<0.05)$. 


\section{Characteristics}

Participant characteristics included age, ankle dorsiflexion strength, disease duration, and MoCA. Greater disease severity was predicted from longer disease duration $\left(R^{2}=0.36\right.$; DW $=2.2 ; p=0.008$; Fig. $6 A)$. Age, strength, and cognitive ability did not relate to disease severity.

\section{Endpoint performance}

Endpoint performance was quantified with force error, temporal error, force variability, and temporal variability. Greater disease severity was predicted only from temporal variability $\left(R^{2}=\right.$ 0.39; DW $=1.9 ; p=0.006$; Fig. $6 B)$. The model suggested that the lesser the temporal variability (part $r=-0.66$ ) the greater the disease severity. Temporal variability was not associated with disease duration $\left(R^{2}=0.1\right.$; $p=0.19)$.

\section{Cerebellar GM}

We quantified cerebellar GM volume in lobule I-IV, V, VI, CrusI, CrusII, VIIb, VIIIa, VIIIb, IX, X and vermis lobules VI, CrusI, CrusII, VIIb, VIIIa, VIIIb, IX, X. Greater disease severity was predicted from vermis $\mathrm{X}$ and cerebellar lobule VIIIb $\left(R^{2}=0.79\right.$; $\mathrm{DW}=1.4 ; p<0.001$; Fig. $6 C)$. The model, suggested that the lesser the GM in Vermis X (part $r=-0.71$ ) and cerebellar lobule VIIIb (part $r=-0.37$ ) the greater the disease severity. Therefore, greater disease severity as measured with ICARS is associated with longer disease duration, lower temporal variability, and lower GM volume in vermis X and cerebellar lobule VIIIb.

\section{Discussion}

In this study, we hypothesized that SCA6 individuals with reduced temporal variability will exhibit smaller cerebellar lobule VI and reduced disease severity. Our results partially support this hypothesis because SCA6 individuals with reduced GM volume of cerebellar lobule VI exhibit reduced temporal variability during fast goal-directed contractions. In contrast to our hypothesis, we found that temporally invariant SCA6 exhibited greater disease severity. Thus, we provide novel evidence that temporal invariance in SCA6 is associated with structural change in cerebellar lobule VI and greater disease severity.

\section{SCA6 and temporal control}

Dysmetria is a cardinal symptom of SCA6, and is defined as the inability to perform accurate goal-directed actions (Manto, 2009; Solodkin and Gomez, 2012). Here, we provide evidence that during fast goal directed actions of the ankle the lower temporal error in SCA6 relates to lower temporal variability. The reduction in temporal variability was driven by $\sim 60 \%$ of SCA6 individuals (SCA6a), who exhibited temporal variability below healthy control levels. The remaining SCA6 individuals (SCA6b) exhibited temporal variability similar to healthy controls.

While many studies in movement disorder pathology find increased motor variability (Hore et al., 2002; Stergiou and Decker, 2011; Smits-Engelsman and Wilson, 2013; Moon et al., 2016), here we observe a unique group of patients that have reduced temporal variability. Thus we addressed the following question: "Does this decrease in temporal variability relate to
B

C

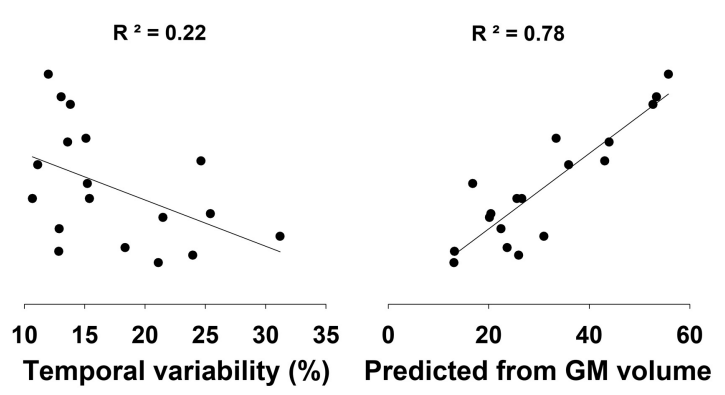
SCA6?"

We provide novel evidence that reduced temporal variability is likely a signature for a specific cerebellar attribute. Indeed, we investigated the relation between GM volume of 17 distinct cerebellar regions (lobules I-IV, V, VI, CrusI, CrusII, VIIb, VIIIa, VIIIb, IX, X and vermis, CrusI, CrusII, VIIb, VIIIa, VIIIb, IX, X) and found that SCA6 individuals with lower GM volume in cerebellar lobule VI exhibited reduced temporal variability. This was specific to lobule VI as no other region analyzed showed association with temporal variability. Although the finding of an association between temporal invariance and reduced GM in cerebellar lobule VI is novel for SCA6, it is consistent with findings from functional imaging that associate this area with timing (Thaut et al., 2009; E et al., 2014). Also, it is indirectly supported by recent findings showing that healthy trained musicians with smaller cerebellar lobule VI exhibit reduced temporal variability (Baer et al., 2015). How can a reduction of GM in lobule VI be associated with better performance in healthy musicians and worse disease severity in SCA6?

Coordination in motor actions results from the performance (amplitude and duration) of individual components and the relative timing among those components (e.g., one joint in a multijoint action) (Dounskaia, 2010). One possibility is that lobule VI is important for the temporal control of individual components, whereas the coordination between individual components is dependent on additional regions of the cerebellum and the CNS (D'Angelo, 2018). Given that the both the CNS and the cerebellum are intact in healthy musicians, the reduced variability of the individual components due to a selective reduction in size of lobule VI, likely enhances the coordination to achieve motor proficiency. In contrast, ataxia results in pancerebellar degeneration with concomitant changes in other brain areas (Kang et al., 2017; Therrien and Bastian, 2019), affecting the timing of motor actions (Ivry and Keele, 1989; Spencer et al., 2003). Indeed, the loss of motor control is likely affected by the loss of Purkinje cells in additional areas of the cerebellum, as we demonstrate with the prediction of disease severity using the ICARS (Fig. 6C). We hypothesize that the additional loss of Purkinje cells in cerebellar lobule VI, which relates to temporal invariance of individual components, reduces the flexibility of individual components to adapt to different task demands and achieve a coordinated output. This finding supports theoretical work suggesting that the loss of degrees of freedom (e.g., temporal invariance of 
individual motor components) reduces redundancy and consequently the ability to exert the same outcome using different combinations (Latash, 2012).

The effect of temporal invariance of individual components on the performance of complex motor tasks remains to be resolved in future studies. Regardless, here, we provide evidence that reduced temporal variability is detrimental to motor function in SCA6. Indeed, we show that SCA6 individuals with reduced temporal variability exhibit greater disease severity, as quantified with ICARS, a functional test of gait, balance, limb control, eye control, and speech. In conclusion, our results provide novel evidence that reduced temporal variability in SCA6 relates to cerebellar deficits and functional impairments. In addition to their clinical significance, these findings have theoretical importance as they propose that reduction of motor variability below the level of healthy controls can be pathological.

\section{Time invariance in SCA6: a distinct phenotype}

First, temporal invariance should not be confused with motor stereotypy. Motor stereotypies (also called stereotypic movement disorder), are rhythmic, fixed, but purposeless movements (Péter et al., 2017), which occur in many disorders including autism and down syndrome (Sanders et al., 2015). Rather, temporal invariance appears to be a limitation of the CNS in planning muscle contraction. As discussed above, this temporal invariance relates to structural degeneration of the cerebellum and specifically loss of GM integrity of cerebellar lobule VI. An important but unresolved question is the following: "Do SCA6 individuals who exhibit temporal invariance reflect a distinct phenotype?"

There are two main arguments supporting this hypothesis. First, SCA6 individuals with temporal invariance exhibit greater disease severity, as evidenced from greater clinical impairment scores (ICARS; Fig. 4). These findings did not relate to differences in age, cognitive capability, or disease duration (Fig. 4). The second argument for a likely distinct phenotype of SCA6 comes from the finding that the temporally invariant SCA6 patients exhibit a specific regional loss of GM in cerebellar lobule VI relative to SCA6 patients that exhibit close to normal levels of temporal variability. Taking these findings together, we propose that some SCA6 individuals may experience an exaggerated loss of GM in lobule VI as a distinct phenotype resulting in temporal invariance in fast single joint contractions and increased functional impairement.

\section{Cerebellar subregions and clinical scores of ataxia}

Greater disease severity was predicted $\left(R^{2}=0.79\right)$ from vermis X (part $r=-0.71$ ) and CL-VIIIb (part $r=-0.37$ ) indicating that reduction of these two areas associates with greater disease severity. First, the prediction of greater disease severity with reduction of Vermis $\mathrm{X}$ is consistant with deficits within the vestibulooccular cerebellum, which is found in the flocculonodular lobe (Stoodley and Schmahmann, 2010). This area receives vestibular and visual information and is involved with balance, vestibular reflexes, and eye movements (Schniepp et al., 2017). Our study, therefore, conforms with classical findings that in cerebellar ataxia, lesions of the vestibulocerebellum cause balance and gait deficits. Second, our predictive model also includes CL-VIII. CL-VIII has been described as the second area of motor representation and together with lobules IX/X (third nonmotor representation) is thought to be involved in the integration of vestibulo/ visuo-motor information with respect to effectors (Guell et al., 2018). Our predictive model for ICARS did not include CL-VI, despite the fact that reduced CL-VI associated with greater dis- ease severity $\left(R^{2}=0.3\right)$. This is possibly related to the fact that ICARS is biased toward overt vestibulo-occular related gait, balance and limb control deficits.

\section{Intragroup separation and hetereogeneity within SCA6}

In our study we show that temporal variability during fast goal directed ankle dorsiflexion distinguishes between two SCA6 groups, one with low variability and one with variability comparable to HC. Interestingly, another study suggested that SCA6 patients may be subdivided into two separate groups, with the difference that separation was based on cognitive performance. Specifically, both SCA6 subgroups showed impairment in visuospatial performance executive functions, but only one group showed additional deficits in mental flexibility/response inhibition and verbal processes (Rentiya et al., 2018).

The presence of subgroups within SCA6 might explain why some studies have found contradictory results about the presence of cognitive deficits in SCA6 (Globas et al., 2003; Suenaga et al., 2008; Rentiya et al., 2018). Similarly, the presence of subtypes based on temporal variability could explain the apparent discrepancy between the result of this study, which shows that ICARS relates to temporal variability, and our previous study which shows that ICARS relates to force error (Casamento-Moran et al., 2015). However, currently we cannot distinguish between the former hypothesis, and the possibility that the difference could be explained by the greater number of participants in this study (19 vs 10). Additional studies including a greater number of subjects are needed to evaluate the reason(s) for intragroup separation and establishing whether hetereogeneity among subjects should be considered in study design and analysis in SCA6.

\section{What is the source of low variability in the SCA6a subgroup?} We found that reduced temporal variability during a single joint contraction relates to the loss of GM in CL-VI. This reduction in variability could be the result of reduced processing capacity at the level of CL-VI. The relation between motor expertise and smaller brain volumes in sensorimotor areas has been postulated to result from pruning of neurons and the positive selection of those responsible for more efficient motor control (James et al., 2014). In contrast, the nonselective pruning resulting from pathological degeneration, as it occurs in SCA6, would result in reduced capacity and suboptimal neural processes. One possibility is that CL-VI degeneration could limit the ability of SCA6a to simultaneously control force and time during this task (similar to a dual-task paradigm). We do not favor this hypothesis because if it were the case, one would expect SCA6a to reduce temporal variability at the expense of increased force variability. However, we find that force variability was similar for SCA6a, SCA6b, and healthy controls. Thus, we hypothesize that reduced temporal variability in SCA6a reflects their reduced capacity to voluntarily modulate the duration of the force contraction. Another possibility is that the exacerbated loss of GM in CL-VI results in impaired motor adaptation leading in overall temporal invariance for SCA6a (Bernard and Seidler, 2013). This would require that SCA6a exhibit lesser temporal variability only at the initial trials and the two groups have similar variability at the end of the acquisition phase. Based on our experiment, the adaptation across 50 trials is not different for the two SCA6 groups (not significant group $\times$ block interaction); thus, we cannot attribute the temporal invariance of SCA6a to impaired motor adaptation. Nonetheless, future studies are needed to understand the involvement of CL-VI in motor control/learning of fast goal directed contractions, by increasing the number of trials, selectively 
lifting the requirements of a force target, and/or, by varying the temporal target.

\section{Conclusion}

For the first time in the SCA literature, we provide evidence that approximately half of SCA6 individuals in this study exhibit lower temporal variability than healthy controls during fast goaldirected contractions. In SCA6 this reduced temporal variability relates to reduced GM volume of cerebellar lobule VI and greater disease severity. It remains unclear whether the temporally invariant SCA6 forms a genetically distinct subtype of SCA6 that would possibly require alternative rehabilitation. In addition, our findings provide novel evidence relevant to theoretical motor control. We argue that pathological motor variability can manifest not only as an exacerbation but also as a reduction relative to healthy controls.

\section{References}

Babin-Ratté S, Sirigu A, Gilles M, Wing A (1999) Impaired anticipatory finger grip-force adjustments in a case of cerebellar degeneration. Exp Brain Res 128:81-85.

Baer LH, Park MT, Bailey JA, Chakravarty MM, Li KZ, Penhune VB (2015) Regional cerebellar volumes are related to early musical training and finger tapping performance. Neuroimage 109:130-139.

Bares M, Apps R, Avanzino L, Breska A, D’Angelo E, Filip P, Gerwig M, Ivry RB, Lawrenson CL, Louis ED, Lusk NA, Manto M, Meck WH, Mitoma H, Petter EA (2019) Consensus paper: Decoding the Contributions of the Cerebellum as a Time Machine. From Neurons to Clinical Applications. Cerebellum 18:266-286.

Bastian AJ, Martin TA, Keating JG, Thach WT (1996) Cerebellar ataxia: abnormal control of interaction torques across multiple joints. J Neurophysiol 76:492-509.

Beck AT, Ward CH, Mendelson M, Mock J, Erbaugh J (1961) An inventory for measuring depression. Arch Gen Psychiatry 4:561-571.

Becker WJ, Kunesch E, Freund HJ (2015) Coordination of a multi-joint movement in normal humans and in patients with cerebellar dysfunction. Can J Neurol Sci 17:264-274.

Benjamini Y, Hochberg Y (1995) Controlling the false discovery rate: a practical and powerful approach to multiple testing. Journal of the Royal Statistical Society Series B (Methodological) 57:289-300.

Bernard JA, Seidler RD (2013) Cerebellar contributions to visuomotor adaptation and motor sequence learning: an ALE meta-analysis. Front Hum Neurosci 7:27.

Carlton LG (1981) Visual information: the control of aiming movements. Q J Exp Psychol A 33:87-93.

Casamento-Moran A, Chen YT, Kwon M, Snyder A, Subramony SH, Vaillancourt DE, Christou EA (2015) Force dysmetria in spinocerebellar ataxia 6 correlates with functional capacity. Front Hum Neurosci 9:184.

D’Angelo E (2018) Physiology of the cerebellum. Handb Clin Neurol 154: 85-108.

Desmurget M, Grafton S (2003) Feedback or feedforward control: end of a dichotomy. In: Taking action: cognitive neuroscience perspectives on intentional acts (Johnson-Frey SH, ed), pp. 289-338. Cambridge, MA: MIT.

Diedrichsen J (2006) A spatially unbiased atlas template of the human cerebellum. Neuroimage 33:127-138.

Dounskaia N (2010) Control of human limb movements: the leading joint hypothesis and its practical applications. Exerc Sport Sci Rev 38:201-208.

E KH, Chen SH, Ho MH, Desmond JE (2014) A meta-analysis of cerebellar contributions to higher cognition from PET and fMRI studies. Hum Brain Mapp 35:593-615.

Elias LJ, Bryden MP, Bulman-Fleming MB (1998) Footedness is a better predictor than is handedness of emotional lateralization. Neuropsychologia 36:37-43.

Globas C, Bösch S, Zühlke Ch, Daum I, Dichgans J, Bürk K (2003) The cerebellum and cognition: intellectual function in spinocerebellar ataxia type 6 (SCA6). J Neurol 250:1482-1487.

Grafton ST, Tunik E (2011) Human basal ganglia and the dynamic control of force during on-line corrections. J Neurosci 31:1600-1605.
Green SB, Salkind NJ (2004) Using SPSS for windows and macintosh: analyzing and understanding, Ed 4. Upper Saddle River, NJ: Prentice Hall.

Grube M, Cooper FE, Chinnery PF, Griffiths TD (2010) Dissociation of duration-based and beat-based auditory timing in cerebellar degeneration. Proc Natl Acad Sci U S A 107:11597-11601.

Guell X, Schmahmann JD, Gabrieli J, Ghosh SS (2018) Functional gradients of the cerebellum. eLife 7:e36652.

Holmes G (1917) The symptoms of acute cerebellar injuries due to gunshot injuries. Brain 40:461-535.

Holmes G (1922) Clinical symptoms of cerebellar disease and their interpretation: the Croonian lecture III. Lancet 2:59-65.

Hore J, Watts S (2005) Timing finger opening in overarm throwing based on a spatial representation of hand path. J Neurophysiol 93:3189-3199.

Hore J, Timmann D, Watts S (2002) Disorders in timing and force of finger opening in overarm throws made by cerebellar subjects. Ann N Y Acad Sci 978:1-15.

Hore J, O'Brien M, Watts S (2005) Control of joint rotations in overarm throws of different speeds made by dominant and nondominant arms. J Neurophysiol 94:3975-3986.

Ivry RB, Keele SW (1989) Timing functions of the cerebellum. J Cogn Neurosci 1:136-152.

James CE, Oechslin MS, Van De Ville D, Hauert CA, Descloux C, Lazeyras F (2014) Musical training intensity yields opposite effects on grey matter density in cognitive versus sensorimotor networks. Brain Struct Funct 219:353-366.

Kang N, Christou EA, Burciu RG, Chung JW, DeSimone JC, Ofori E, Ashizawa T, Subramony SH, Vaillancourt DE (2017) Sensory and motor cortex function contributes to symptom severity in spinocerebellar ataxia type 6. Brain Struct Funct 222:1039-1052.

Latash ML (2012) The bliss (not the problem) of motor abundance (not redundancy). Exp Brain Res 217:1-5.

Manto M (2009) Mechanisms of human cerebellar dysmetria: experimental evidence and current conceptual bases. J Neuroeng Rehabil 6:10.

Moon Y, Sung J, An R, Hernandez ME, Sosnoff JJ (2016) Gait variability in people with neurological disorders: a systematic review and metaanalysis. Hum Mov Sci 47:197-208.

Nasreddine ZS, Phillips NA, Bédirian V, Charbonneau S, Whitehead V, Collin I, Cummings JL, Chertkow H (2005) The montreal cognitive assessment, MoCA: a brief screening tool for mild cognitive impairment. J Am Geriatr Soc 53:695-699.

Nowak DA, Hermsdörfer J, Marquardt C, Fuchs HH (2002) Grip and load force coupling during discrete vertical arm movements with a grasped object in cerebellar atrophy. Exp Brain Res 145:28-39.

Oostwoud Wijdenes L, Brenner E, Smeets JB (2011) Fast and fine-tuned corrections when the target of a hand movement is displaced. Exp Brain Res 214:453-462.

Paquette S, Fujii S, Li HC, Schlaug G (2017) The cerebellum's contribution to beat interval discrimination. Neuroimage 163:177-182.

Péter Z, Oliphant ME, Fernandez TV (2017) Motor stereotypies: a pathophysiological review. Front Neurosci 11:171.

Prablanc C, Martin O (1992) Automatic control during hand reaching at undetected two-dimensional target displacements. J Neurophysiol 67: $455-469$

Rentiya ZS, Jung BC, Bae J, Liszewski CM, Fishman A, Du AX, Margolis RL, Ying SH (2018) Selective patterns of cognitive impairment in spinocerebellar ataxia type 6 and idiopathic late-onset cerebellar ataxia. Arch Clin Neuropsychol 33:427-436.

Sanders SJ, He X, Willsey AJ, Ercan-Sencicek AG, Samocha KE, Cicek AE, Murtha MT, Bal VH, Bishop SL, Dong S, Goldberg AP, Jinlu C, Keaney JF 3rd, Klei L, Mandell JD, Moreno-De-Luca D, Poultney CS, Robinson EB, Smith L, Solli-Nowlan T (2015) Insights into autism spectrum disorder genomic architecture and biology from 71 risk loci. Neuron 87:12151233.

Saunders JA, Knill DC (2004) Visual feedback control of hand movements. J Neurosci 24:3223-3234

Saute JA, Donis KC, Serrano-Munuera C, Genis D, Ramirez LT, Mazzetti P, Pérez LV, Latorre P, Sequeiros J, Matilla-Dueñas A, Jardim LB; Iberoamerican Multidisciplinary Network for the Study of Movement Disorders (RIBERMOV) Study Group. (2012) Ataxia rating scales-psychometric profiles, natural history and their application in clinical trials. Cerebellum 11:488-504. 
Schniepp R, Möhwald K, Wuehr M (2017) Gait ataxia in humans: vestibular and cerebellar control of dynamic stability. J Neurol 264:87-92.

Smits-Engelsman BC, Wilson PH (2013) Noise, variability, and motor performance in developmental coordination disorder. Dev Med Child Neurol 55:69-72.

Solodkin A, Gomez CM (2012) Spinocerebellar ataxia type 6. Handb Clin Neurol 103:461-473.

Spencer RM, Zelaznik HN, Diedrichsen J, Ivry RB (2003) Disrupted timing of discontinuous but not continuous movements by cerebellar lesions. Science 300:1437-1439.

Stergiou N, Decker LM (2011) Human movement variability, nonlinear dynamics, and pathology: is there a connection? Hum Mov Sci 30:869-888.

Stoodley CJ, Schmahmann JD (2010) Evidence for topographic organization in the cerebellum of motor control versus cognitive and affective processing. Cortex 46:831-844.

Storey E, Tuck K, Hester R, Hughes A, Churchyard A (2004) Inter-rater reliability of the international cooperative ataxia rating scale (ICARS). Mov Disord 19:190-192.

Suenaga M, Kawai Y, Watanabe H, Atsuta N, Ito M, Tanaka F, Katsuno M, Fukatsu H, Naganawa S, Sobue G (2008) Cognitive impairment in spinocerebellar ataxia type 6. J Neurol Neurosurg Psychiatry 79:496-499.
Thaut MH, Stephan KM, Wunderlich G, Schicks W, Tellmann L, Herzog H, McIntosh GC, Seitz RJ, Hömberg V (2009) Distinct cortico-cerebellar activations in rhythmic auditory motor synchronization. Cortex 45:44-53.

Therrien AS, Bastian AJ (2019) The cerebellum as a movement sensor. Neurosci Lett 688:37-40.

Topka H, Konczak J, Dichgans J (1998) Coordination of multi-joint arm movements in cerebellar ataxia: analysis of hand and angular kinematics. Exp Brain Res 119:483-492.

Trouillas P, Takayanagi T, Hallett M, Currier RD, Subramony SH, Wessel K, Bryer A, Diener HC, Massaquoi S, Gomez CM, Coutinho P, Ben Hamida M, Campanella G, Filla A, Schut L, Timann D, Honnorat J, Nighoghossian N, Manyam B (1997) International cooperative ataxia rating scale for pharmacological assessment of the cerebellar syndrome. the ataxia neuropharmacology committee of the world federation of neurology. J Neurol Sci 145:205-211.

Zhuchenko O, Bailey J, Bonnen P, Ashizawa T, Stockton DW, Amos C, Dobyns WB, Subramony SH, Zoghbi HY, Lee CC (1997) Autosomal dominant cerebellar ataxia (SCA6) associated with small polyglutamine expansions in the alpha 1A-voltage-dependent calcium channel. Nat Genet 15:62-69. 